\title{
Commentary: Regulation of autoimmune arthritis by the SHP-1 tyrosine phosphatase
}

\author{
Adrienn Markovics ${ }^{1 *}$, Daniel M. Toth ${ }^{2}$, Tibor T. Glant ${ }^{1}$, Katalin Mikecz' \\ 1Department of Orthopedic Surgery, Rush University Medical Center, Chicago, IL, USA \\ 2Division of Rheumatology, Department of Medicine, Icahn School of Medicine at Mount Sinai, New York, NY, USA
}

Article Info

\section{Article Notes}

Received: April 28, 2021

Accepted: June 01, 2021

\section{*Correspondence:}

*Dr. Adrienn Markovics, Department of Orthopedic Surgery,

Rush University Medical Center, Chicago, IL, USA;

Email: Adrienn_Markovics@rush.edu.

${ }^{\circ} 2021$ Markovics A. This article is distributed under the terms of the Creative Commons Attribution 4.0 International License.

\section{Keywords}

Rheumatoid arthritis

Autoimmunity

Tyrosine phosphatase

T cells

Proteoglycan-induced arthritis

SHP-1

\section{Abstract}

Rheumatoid arthritis (RA) is a systemic autoimmune disease leading primarily to the inflammatory destruction of the synovial joints, ultimately resulting in loss of mobility and a decreased quality of life. Recent advances in RA-related research resulted in the introduction of Janus kinase (JAK) inhibitors to the therapeutic arsenal. JAK inhibitors are orally available and provide efficacy similar to that of disease-modifying anti-rheumatic drugs. However, due to the limited selectivity of these drugs, many RA patients experience adverse effects. The potential benefit of modulating protein tyrosine phosphatases, instead of inhibiting kinases, has not been explored. Using an animal model of RA, our group investigated the role of the Src homology region 2 domain-containing phosphatase 1 (SHP-1) in inflammatory arthritis. The purpose of the present article is to highlight important conclusions of our previous paper entitled "Regulation of autoimmune arthritis by the SHP-1 tyrosine phosphatase". Herein we briefly present and discuss the observations of this study. We also outline future directions toward investigating the therapeutic potential of targeting SHP-1 in inflammatory arthritis in order to develop a new type of orally available drugs for the treatment of RA.

\section{Introduction}

The goal of this Commentary is to provide information about our recently published article "Regulation of autoimmune arthritis by the SHP-1 tyrosine phosphatase"1 and to highlight the relevance of our findings to arthritis-focused research and therapy. The Src homology region 2 domain-containing phosphatase 1 (SHP-1) is a non-receptor protein tyrosine phosphatase, expressed mainly in the cytoplasm of hematopoietic cells ${ }^{2}$. It has two Src homology region 2 (SH2) domains that bind to receptors containing immunoreceptor tyrosine-based inhibition motifs (ITIMs) while the catalytic domain dephosphorylates tyrosine residues within the substrate ${ }^{3}$. In an inactive state, SHP-1 is in an auto inhibitory conformation, as the $\mathrm{SH} 2$ domains fold over the catalytic domain and prevent enzymatic activity ${ }^{4}$. SHP-1 exerts a negative regulatory effect on both adaptive and innate immune cells ${ }^{5}$. Regorafenib, a small-molecule, orally available "multi kinase inhibitor" has been shown to directly activate the phosphatase activity of SHP-1 by insertion between the $\mathrm{SH} 2$ and catalytic domains of the enzyme, thereby relieving its auto inhibitory conformation ${ }^{6}$. Regorafenib is currently considered as a therapeutic agent for metastatic colorectal cancer due to its ability to block several protein kinases involved in oncogenesis ${ }^{7,8}$. Another kinase inhibitor, nintedanib, has been also shown to directly activate SHP-1 and induce apoptosis in triple negative breast cancer cells 9 . To our knowledge, the effects of regorafenib treatment on autoimmune conditions have not been investigated before. 
In therapeutic approaches to autoimmune diseases such as rheumatoid arthritis (RA), there is an ongoing effort to identify small-molecule drugs capable of intercepting signal transduction checkpoints in immune cells, thus abrogating the inflammatory process. Immune homeostasis requires the finely-tuned and coordinated function of protein kinases and phosphatases. Kinase inhibitors presented a breakthrough in the therapy of RA in the last decade. Pan-Janus kinase (JAK) inhibitors such as tofacitinib can block the signaling pathways of a variety of cytokines and are popular oral treatment options in $\mathrm{RA}^{10}$. However, broad cytokine inhibition may lead to adverse and/or off-target effects including infection, cytopenias, and potentially, the development of malignancies in the long term ${ }^{11}$. Highly selective JAK inhibition could increase the efficacy and safety profile of these drugs. Contrary to protein kinases, less is known about the possible therapeutic potential of modulating protein phosphatases. For a long time they have been considered difficult drug discovery targets owing to their evolutionary diversity, the conserved active sites within the families, and the difficulty to assign substrates to specific phosphatases ${ }^{12}$. However, recent development in the approaches to studying them resulted in renewed attention towards protein phosphatases in drug discovery programs.

Our research group has a history of studying the role of SHP-1. Spontaneously arising mutations in the Shp1 gene cause reduced or absent catalytic function of the enzyme, leading to the so-called motheaten (me) phenotype in mice, and may also contribute to neutrophilic dermatoses in humans ${ }^{13}$. Me mice exhibit severe skin inflammation and systemic autoimmunity ${ }^{14}$. Our group described a condition caused by the spontaneous insertion of a B2 element in the $\operatorname{Shp} 1$ gene, leading to me-like phenotype (meB2) ${ }^{15}$. The limited amount of enzymatically active SHP-1 in meB2 mice resulted in delayed apoptosis and altered function of neutrophils. The autoinflammatory symptoms in meB2 mice were found to be milder than in me mice with other mutations of the gene, and shared some features with human neutrophilic dermatosis ${ }^{15}$.

\section{Results and Discussion}

To rescue the highly inflammatory phenotype seen in meB2 mice, we generated Shp1-transgenic mice overexpressing the enzyme, and intercrossing the meB2 with homozygous Shp1-transgenic mice resulted in a normal phenotype (unpublished results). Since the role of SHP-1 in RA or animal models of inflammatory arthritis has not been investigated before, we then backcrossed the transgenic mice to the proteoglycan-induced arthritis (PGIA) susceptible BALB/c genetic background. Mice heterozygous for the Shp1 transgene showed a 6-fold increase in Shp1 gene expression and 3.7-times higher enzymatic activity compared to wild type (WT) BALB/c mice, but they were lower than the levels of transgene expression and enzyme activity in the homozygous transgenic line ${ }^{1}$. B cells from naïve mice of both transgenic lines responded with significantly lower proliferation to in vitro anti-IgM stimulation. T cells of naïve homozygous transgenic mice proliferated less in response to polyclonal (anti-CD3/CD28) stimulation than T cells from WT mice, while the proliferation of heterozygous transgenic T cells did not differ significantly from that of WT cells.

When PGIA was induced in both transgenic genotypes, we found that mice homozygous for the Shp1 transgene were almost fully resistant to the development of PGIA, while those with heterozygous transgene expression showed intermediate arthritis incidence and severity. Due to their moderate susceptibility to PGIA, we selected the heterozygous transgenic line for further investigation into the negative effects of SHP- 1 on immune cell signaling, and whether these effects can be exploited to halt autoimmune joint disease. Phosphorylation-specific protein microarray analysis suggested that reduced immune cell activation and milder arthritis in the presence of slight SHP-1 overexpression could be attributed to the impairment of tyrosine phosphorylation of key signaling elements of the JAK/STAT pathway. Next, we treated WT mice orally with regorafenib to pharmacologically activate SHP1 in two different settings of PGIA development. When administered before disease onset, regorafenib was able to diminish PGIA incidence and severity; when the treatment began at an early stage of PGIA, the drug reduced arthritis severity. However, it is important to note that regorafenib may not be specific for SHP-1, but can also activate other protein tyrosine phosphatases such as the Src homology region 2 domain-containing phosphatase 2 (SHP-2). SHP-2 has a broader substrate repertoire than SHP- $1^{16}$, therefore, it is conceivable that regorafenib might have off-target effects or might be toxic to cells. Indeed, we observed some weight loss in the group of mice receiving a high dose $(20 \mathrm{mg} / \mathrm{kg}$ body weight) regorafenib. Since SHP-1 can act on multiple cell types and might affect the activity of different phosphatases, there is a need to develop new small-molecule drugs that are more selective activators of SHP-1 than regorafenib, in order to explore their potential to antagonize inflammatory arthritis without serious side effects.

\section{Future Directions}

Due to the differences between the mouse and human immune systems (including those involving both adaptive and innate immune cells $)^{17}$, the direct translation of our results to the human system is problematic. Our current aim is to establish a novel, humanized PGIA model and to investigate if SHP-1 activation has a therapeutic effect on PGIA (or other forms of inflammatory arthritis) in 
humanized mice. Human immune system (HIS) mice are immunodeficient mice reconstituted with human hematopoietic stem cells, which provide an excellent translational research $\operatorname{tool}^{18}$. Several humanized mouse models have been established to study autoimmune conditions such as RA. While all of these models have limitations, HIS mice provide the most advanced animalhuman chimeras for preclinical investigations in RA, as discussed in ${ }^{19}$. Exploring the potential therapeutic value of SHP-1 activation in HIS mice would be of great importance, as these mice could represent a direct translational bridge between the human and mouse immune systems. On the basis of our previous observations, we firmly believe that establishing a humanized PGIA model and exploring the effects of protein tyrosine phosphatase activator treatment in mice reconstituted with human immune cells would provide a powerful preclinical tool for translational studies. Furthermore, if successful, the results of such experiments would enable us to conduct, in collaboration with drug discovery experts, basic and preclinical studies focusing on the development of new, orally available and selective activators of SHP-1.

\section{Funding}

The authors were supported by a Musculoskeletal Research Pilot Project grant from the Rush Translational Sciences Consortium (to Markovics A) and by a grant from the NIH/NIAMS (R01 AR064206-05, to Mikecz K).

\section{Conflict of Interest}

The authors declare that they have no conflict of interest.

\section{References}

1. Markovics A, Toth DM, Glant TT, et al. Regulation of autoimmune arthritis by the SHP-1 tyrosine phosphatase. Arthritis research \& therapy. 2020; 22(1): 160

2. Neel BG, Tonks NK. Protein tyrosine phosphatases in signal transduction. Current opinion in cell biology. 1997; 9(2): 193-204.

3. Coggeshall KM, Nakamura K, Phee H. How do inhibitory phosphatases work? Molecular immunology. 2002; 39(9): 521-9.

4. Martin A, Tsui HW, Shulman MJ, et al. Murine SHP-1 splice variants with altered Src homology 2 (SH2) domains. Implications for the
SH2-mediated intramolecular regulation of SHP-1. The Journal of biological chemistry. 1999; 274(31): 21725-34.

5. Garg M, Wahid M, Khan FD. Regulation of peripheral and central immunity: Understanding the role of Src homology 2 domaincontaining tyrosine phosphatases, SHP-1 \& SHP-2. Immunobiology. $2020 ; 225(1): 151847$

6. Fan LC, Teng HW, Shiau CW, et al. SHP-1 is a target of regorafenib in colorectal cancer. Oncotarget. 2014; 5(15): 6243-51.

7. Wilhelm SM, Dumas J, Adnane L, et al. Regorafenib (BAY 73-4506): a new oral multikinase inhibitor of angiogenic, stromal and oncogenic receptor tyrosine kinases with potent preclinical antitumor activity. International journal of cancer. 2011; 129(1): 245-55.

8. Majithia N, Grothey A. Regorafenib in the treatment of colorectal cancer. Expert opinion on pharmacotherapy. 2016; 17(1): 137-45.

9. Liu CY, Huang TT, Chu PY, et al. The tyrosine kinase inhibitor nintedanib activates SHP-1 and induces apoptosis in triple-negative breast cancer cells. Experimental \& molecular medicine. 2017; 49(8): e366.

10. Dhillon S. Tofacitinib: A Review in Rheumatoid Arthritis. Drugs. 2017; 77(18): 1987-2001.

11. Gadina M, Le MT, Schwartz DM, et al. Janus kinases to jakinibs: from basic insights to clinical practice. Rheumatology. 2019; 58(Suppl 1): i4-i16.

12. Kohn M. Turn and Face the Strange: A New View on Phosphatases. ACS central science. 2020; 6(4): 467-77.

13. Nesterovitch AB, Gyorfy Z, Hoffman MD, et al. Alteration in the gene encoding protein tyrosine phosphatase nonreceptor type 6 (PTPN6/ SHP1) may contribute to neutrophilic dermatoses. The American journal of pathology. 2011; 178(4): 1434-41.

14. Tsui HW, Siminovitch KA, de Souza L, et al. Motheaten and viable motheaten mice have mutations in the haematopoietic cell phosphatase gene. Nature genetics. 1993; 4(2): 124-9.

15. Nesterovitch AB, Szanto S, Gonda A, et al. Spontaneous insertion of a b2 element in the ptpn 6 gene drives a systemic autoinflammatory disease in mice resembling neutrophilic dermatosis in humans. The American journal of pathology. 2011; 178(4): 1701-14.

16. Lorenz U. SHP-1 and SHP-2 in T cells: two phosphatases functioning at many levels. Immunological reviews. 2009; 228(1): 342-59.

17. Mestas J, Hughes CC. Of mice and not men: differences between mouse and human immunology. Journal of immunology. 2004; 172(5): 2731-8.

18. Pearson T, Greiner DL, Shultz LD. Creation of "humanized" mice to study human immunity. Current protocols in immunology. 2008; Chapter 15: Unit 15.21.

19. Schinnerling K, Rosas C, Soto L, et al. Humanized Mouse Models of Rheumatoid Arthritis for Studies on Immunopathogenesis and Preclinical Testing of Cell-Based Therapies. Frontiers in immunology. 2019; 10: 203. 